\title{
About solution of multipoint boundary problem of static analysis of deep beam with the use of combined application of finite element method and discrete-continual finite element method. part 1: formulation of the problem and general principles of approximation
}

\author{
Leonid Lyakhovich ${ }^{1}$, Oleg Negrozov ${ }^{2, *}$ \\ ${ }^{1}$ Tomsk State University of Architecture and Building, Department of Structural Mechanics, \\ Solyanaya sq., Tomsk, 634003, Russia \\ ${ }^{2}$ National Research Moscow State University of Civil Engineering, Department of Applied \\ Mathematics; 26, Yaroslavskoe Shosse, Moscow, 129337, Russia
}

\begin{abstract}
This paper is devoted to formulation and general principles of approximation of multipoint boundary problem of static analysis of deep beam with the use of combined application of finite element method (FEM) discrete-continual finite element method (DCFEM). The field of application of DCFEM comprises structures with regular physical and geometrical parameters in some dimension ("basic" dimension). DCFEM presupposes finite element approximation for non-basic dimension while in the basic dimension problem remains continual. DCFEM is based on analytical solutions of resulting multipoint boundary problems for systems of ordinary differential equations with piecewise-constant coefficients.
\end{abstract}

\section{Formulation of the problem}

Let's consider multipoint boundary problem of static analysis of deep beam (Fig. 1) [7, $10-12,14,15,17-23,25,27-32]$. Some elements of notation system is presented at Fig. 1. Corresponding design model is two-dimensional theory of elasticity [9].

Let's $\Omega$ be domain occupied by structure, $\Omega=\left\{\left(x_{1}, x_{2}\right): 0<x_{1}<l_{1}, 0<x_{2}<l_{2}\right\}$ or

$$
\Omega=\bigcup_{k=1}^{n_{b}-1} \Omega_{k}, \quad \Omega_{k}=\left\{\left(x_{1}, x_{2}\right): 0<x_{1}<l_{1, k}, \quad x_{2, k}^{b}<x_{2}<x_{2, k+1}^{b}\right\} ;
$$

$x_{1}, x_{2}$ are coordinates ( $x_{2}$ corresponds to basic dimension); $x_{2, k}^{b}, k=1,2, \ldots, n_{b}$ are coordinates of corresponding boundary points (cross-sections) along basic dimension (for mulipoint boudary peoblems $n_{b}>2 ; \Omega_{k}, k=1,2, \ldots, n_{b}-1$ are subdomains of $\Omega$.

* Corresponding author: genromgsu@gmail.com 


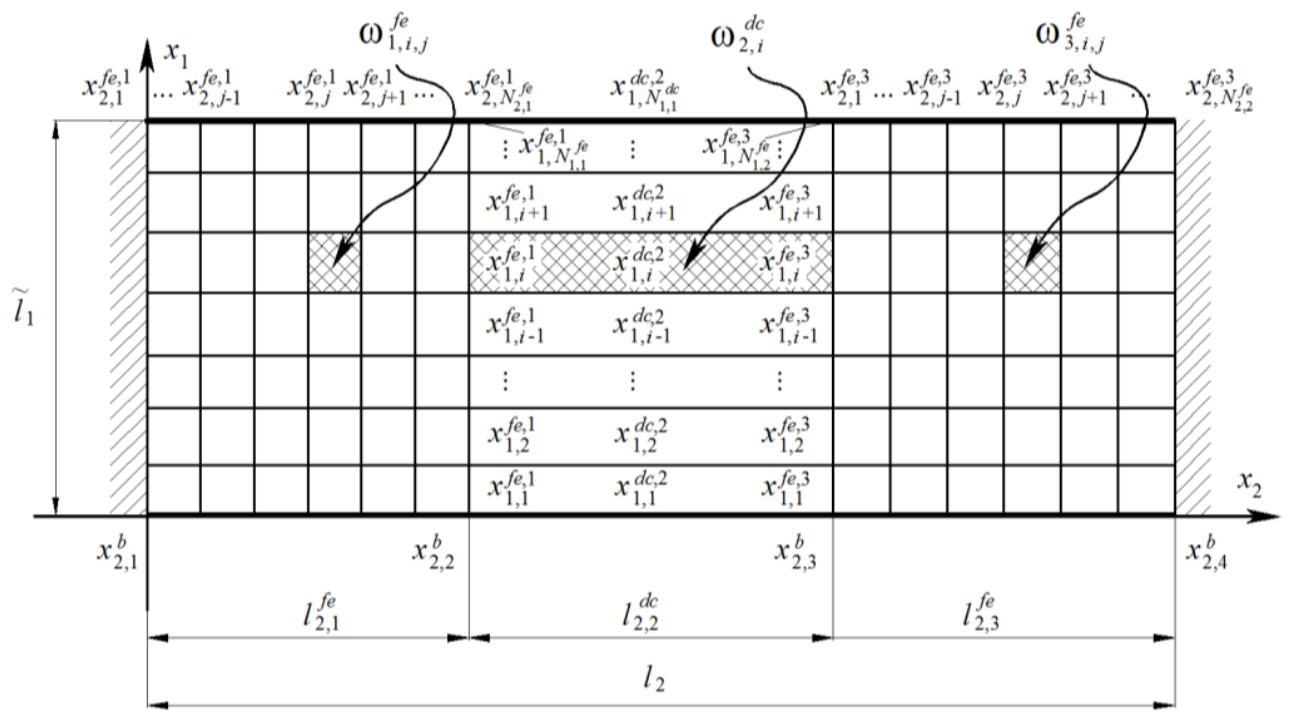

Fig. 1. Sample of three-point boundary problem.

Let $l_{1, k}=$ const for $x_{2, k}^{b}<x_{2}<x_{2, k+1}^{b}$. However in general case $l_{1}=l_{1}\left(x_{2}\right) \neq$ const (parameter $l_{1}$ can be piecewise-constant).

Let $\omega_{k}, k=1,2, \ldots, n_{b}-1$ are extended subdomains, embordering subdomains $\Omega_{k} \subset \omega_{k}, k=1,2, \ldots, n_{b}-1$,

$$
\omega=\bigcup_{k=1}^{n_{b}-1} \omega_{k}, \quad \omega_{k}=\left\{\left(x_{1}, x_{2}\right): 0<x_{1}<\tilde{l}_{1}, \quad x_{2, k}^{b}<x_{2}<x_{2, k+1}^{b}\right\} \supset \Omega_{k} ;
$$

\section{General principles of domain approximation}

Let physical and geometrical parameters within one group of subdomains from (2) is constant in some dimension ("basic" dimension). Thus, it is recommended to use DCFEM $[1-5,30,35]$ for approximation of these subdomains (discrete-continual design model is introduced). Let physical and geometrical parameters within other group of subdomains from (2) is arbitrary varying. FEM [6,8,9,13,24,26,33,34] can be effectively used for approximation here. Thus, combined application of DCFEM and FEM is advisable.

So-called approximation parameter $\rho_{k}$ can be introduced in accordance with the following rule : $\rho_{k}=1$ for approximation with the use of FEM; $\rho_{k}=2$ for approximation with the use of DCFEM .

\section{About numbering of subdomains}

Various approaches can be used for numbering of subdomains.

The first approach provides separate numbering of subdomains with different types of approximation:

$$
k_{1}=k_{1}(k)=\sum_{s=1}^{k}\left|\rho_{s}-2\right| ; \quad k_{2}=k_{2}(k)=\sum_{s=1}^{k}\left|\rho_{s}-1\right|,
$$


where $k$ is initial number of subdomain $\omega_{k} ; k_{1}=k_{1}(k)$ is the corresponding number of subdomain with approximation with the use of FEM; $k_{2}=k_{2}(k)$ is the corresponding number of subdomain with approximation with the use of DCFEM.

Various approaches can be used for numbering of subdomains.

We can certainly construct inverse relationships by tabulating the results of calculations using formulas (3).

$$
k=k\left(k_{1}\right) ; \quad k=k\left(k_{2}\right),
$$

Thus we can rewrite (2) in the following form:

$$
\omega=\bigcup_{k_{1}=1}^{N_{f e}} \omega_{k_{1}}^{f e}+\bigcup_{k_{2}=1}^{N_{d c}} \omega_{k_{2}}^{d c} .
$$

Besides, the following relations are valid:

$$
N_{f e}=\sum_{s=1}^{n_{b}-1}\left|\rho_{s}-2\right| ; \quad N_{d c}=\sum_{s=1}^{n_{b}-1}\left|\rho_{s}-1\right| ; \quad N_{f e}+N_{d c}=n_{b}-1
$$

where $\omega_{k_{1}}^{f e}, k_{1}=1,2, \ldots, N_{f e}$ are subdomain with approximation with the use of FEM ; $\omega_{k_{2}}^{d c}, k_{2}=1,2, \ldots, N_{d c}$ are subdomain with approximation with the use of DCFEM.

The second approach, on the contrary, is based on a linked numbering of subdomains with different types of approximation. Formula (2) can be used, where

$$
\omega_{k}=\left\{\begin{array}{lll}
\omega_{k}^{f e}, & \text { if } & \rho_{k}=1 \\
\omega_{k}^{d c}, & \text { if } & \rho_{k}=2
\end{array}\right.
$$

while formulas (3) and (4) are not required. The second approach is used in this paper.

\section{About numbering of finite elements and discrete-continual finite elements}

Let's consider arbitrary subdomain $\omega_{k}^{f e}$. We can introduce notation

$$
l_{2, k}^{f e}=x_{2, k+1}^{b}-x_{2, k}^{b} \quad \text { if } \quad \rho_{k}=1 \text {. }
$$

Let's $x_{1, i}^{f e, k}, i=1,2, \ldots, N_{1, k}^{f e}$ and $x_{2, j}^{f e, k}, j=1,2, \ldots, N_{2, k}^{f e}$ are coordinates coordinates (along $x_{1}$ and $\left.x_{2}\right)$ of nodes of finite elements, which are used for approximation of domain $\omega_{k}^{f e}$; $\left(N_{1, k}^{f e}-1\right)$ and $\left(N_{2, k}^{f e}-1\right)$ are numbers of finite elements along coordinates $x_{1}$ and $x_{2}$, which are used for approximation of $\omega_{k}^{f e}$. Three-index system is used for numbering of finite elements, which are used for approximation of $\omega_{k}^{f e}$. Typical number of has the form $(k, i, j)$, where $k$ is the number of subdomain with approximation with the use of FEM, $i$ and $j$ are numbers of elements (along $x_{1}$ and $\left.x_{2}\right) ; \omega_{k, i, j}^{f e}$ is corresponding finite element.

Let's consider arbitrary subdomain $\omega_{k}^{d c}$. We can introduce notation 


$$
l_{2, k}^{d c}=x_{2, k+1}^{b}-x_{2, k}^{b} \quad \text { if } \rho_{k}=2 .
$$

Let's $x_{1, i}^{d c, k}, i=1,2, \ldots, N_{1, k}^{d c}$ are coordinates (along $x_{1}$ ) of nodes (nodal lines) of discretecontinual finite elements, which are used for approximation of domain $\omega_{k}^{d c} ;\left(N_{1, k}^{d c}-1\right)$ is the number of discrete-continual finite elements, which are used for approximation of $\omega_{k}^{d c}$. Twoindex notation system is used for numbering of discrete-continual finite elements, which are used for approximation of $\omega_{k}^{d c}$. Typical number of has the form $(k, i)$, where $k$ is the number of subdomain, $i$ is the number of element (along $x_{1}$ ).

We can introduce notation

$$
\begin{gathered}
N_{1, k}=\left\{\begin{array}{ll}
N_{1, k}^{f e} & \text { if } \rho_{k}=1 \\
N_{1, k}^{d c} & \text { if } \rho_{k}=2 ;
\end{array} \quad N_{2, k}=N_{2, k}^{f e} \text { if } \rho_{k}=1 ;\right. \\
x_{1, k, i}=\left\{\begin{array}{lll}
x_{1, i}^{f e, k} & \text { if } \rho_{k}=1 \\
x_{1, i}^{d c, k} & \text { if } \rho_{k}=2 ; & x_{2, k, j}=x_{2, j}^{f e, k} \text { if } \rho_{k}=1 ;
\end{array}\right. \\
l_{2, k}=\left\{\begin{array}{lll}
l_{2, k}^{f f} & \text { if } \rho_{k}=1 \\
l_{2, k}^{d c} & \text { if } \rho_{k}=2 .
\end{array}\right.
\end{gathered}
$$

It should be noted that in the simples cases (such case in considered in the distinctive paper) discretization of structure is constant along $x_{1}$ throughout the domain (otherwise the mathematical constructions given below are substantially more complicated). We have

$$
N_{1, k}=N_{1}, \quad k=1,2, \ldots, n_{b}-1 ; \quad x_{1, k, i}=x_{1, i}, k=1,2, \ldots, n_{b}-1, \quad i=1,2, \ldots, N_{1} .
$$

In should be noted that notation from $[3-5,30]$ is also used in this paper.

\section{Discrete (finite element) approximation model for subdomain}

Let's consider arbitrary subdomain $\omega_{k}^{f e}$. Discrete (finite element) approximation model for the considering two-dimensional problems presupposes finite element approximation along $x_{1}$ and $x_{2}$. Thus extended subdomain $\omega_{k}^{f e}$ is divided into finite elements,

$$
\omega_{k}^{f e}=\bigcup_{i=1}^{N_{1}-1 N_{2, k}-1} \bigcup_{j=1}^{f e} \omega_{k, i, j}^{f e}, \quad \omega_{k, i, j}^{f e}=\left\{\left(x_{1}, x_{2}\right): x_{1, i}<x_{1}<x_{1, i+1}, \quad x_{2, k, j}<x_{2}<x_{2, k, j+1}\right\} .
$$

Lame constants for finite element are defined by formulas:

$$
\bar{\lambda}_{k, i, j}=\theta_{k, i, j} \lambda ; \quad \bar{\mu}_{k, i, j}=\theta_{k, i, j} \mu, \text { where } \theta_{k, i, j}=\left\{\begin{array}{lc}
1, & \omega_{k, i, j}^{f e} \subset \Omega_{k} ; \\
0, & \omega_{k, i, j}^{f e} \not \subset \Omega_{k}
\end{array}\right.
$$

$\theta_{k, i, j}$ is the characteristic function of element $\omega_{k, i, j}^{f e}$. 
Basic nodal unknowns are displacement components $u_{1}^{(k)}, u_{2}^{(k)}$ (superscript “ $(k)$ " hereinafter corresponds to the number of considered subdomain i.e. $\left.\omega_{k}=\omega_{k}^{f e}\right)$. Thus for node $(k, i, j)$ we have the following unknowns: $u_{1}^{(k, i, j)}, u_{2}^{(k, i, j)}$.

Bilinear approximation of unknowns is used within finite element (conventional plane rectangular 4-node finite element of two-dimensional problem of elasticity theory (Fig. 2)).
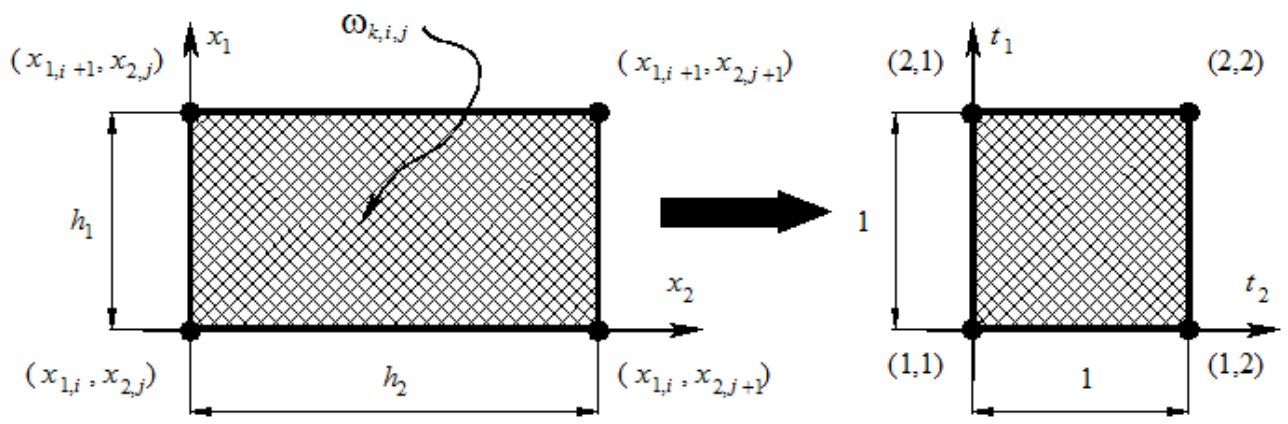

Fig. 2. Finite element and its local coordinate system.

Computing of partial derivatives of displacements, deformations and stresses within the finite element, nodal stresses and nodal deformations with allowance for averaging is described in [3-5,30].

As known, FEM is reduced to the solution of systems of $2 N_{1} N_{2, k}$ linear algebraic equations:

$$
K_{k} \bar{U}_{k}=\bar{R}_{k}
$$

where $\bar{U}_{k}$ is global vector of nodal unknowns (subscript “ $(k)$ " corresponds to the number of subdomain $\omega_{k}=\omega_{k}^{f e}$ ),

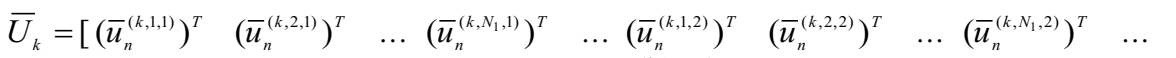

$$
\begin{aligned}
& \left.\ldots\left(\bar{u}_{n}^{\left(k, 1, N_{2, k}\right)}\right)^{T} \quad\left(\bar{u}_{n}^{\left(k, 2, N_{2, k}\right)}\right)^{T} \quad \ldots\left(\bar{u}_{n}^{\left(k, N_{1}, N_{2, k}\right)}\right)^{T}\right]^{T} \text {; } \\
& \bar{u}_{n}^{(k, i, j)}=\left[\begin{array}{ll}
u_{1}^{(k, i, j)} & u_{2}^{(k, i, j)}
\end{array}\right]^{T}, \quad i=1,2, \ldots, N_{1}, \quad j=1,2, \ldots, N_{2, k} ;
\end{aligned}
$$

$K_{k}$ is global stiffness matrix of order $2 N_{1} N_{2, k} ; \bar{R}_{k}$ is global right-side vector of order $2 N_{1} N_{2, k}$ (global load vector).

\section{Discrete-continual approximation model for subdomain}

Let's consider arbitrary subdomain $\omega_{k}^{d c}$. Discrete-continual approximation model is used for two-dimensional problems. It presupposes mesh approximation for non-basic dimension of extended domain (along $x_{1}$ ) while in the basic dimension (along $x_{2}$ ) problem remains continual. Thus subdomain $\omega_{1}$ is divided into discrete-continual finite elements

$$
\omega_{k}^{d c}=\bigcup_{i=1}^{N_{1}-1} \omega_{k, i}^{d c}, \quad \omega_{k, i}^{d c}=\left\{\left(x_{1}, x_{2}\right): x_{1, i}<x_{1}<x_{1, i+1}, \quad x_{2, k}^{b}<x_{2}<x_{2, k+1}^{b}\right\} .
$$


Lame constants for finite element are defined by formulas:

$$
\bar{\lambda}_{k, i}=\theta_{k, i} \lambda ; \quad \bar{\mu}_{k, i}=\theta_{k, i} \mu, \quad \text { where } \theta_{k, i}=\left\{\begin{array}{lc}
1, & \omega_{k, i}^{d c} \subset \Omega_{k} ; \\
0, & \omega_{k, i}^{d c} \not \subset \Omega_{k} ;
\end{array}\right.
$$

$\theta_{k, i}$ is the characteristic function of element $\omega_{k, i}^{d c}$.

Basic nodal unknown functions are displacement components $u_{1}^{(k)}, u_{2}^{(k)}$ and their derivatives $v_{1}^{(k)}, v_{2}^{(k)}$ with respect to $x_{2}$ (superscript " $(k)$ " hereinafter corresponds to the number of considered subdomain i.e. $\left.\omega_{k}=\omega_{k}^{d c}\right)$. Thus for node $(k, i)$ we have the following unknown functions: $u_{1}^{(k, i)}, u_{2}^{(k, i)}$ and $v_{1}^{(k, i)}, v_{2}^{(k, i)}$.

Linear approximation is used for unknown functions within discrete-continual finite element.

DCFEM is reduced at some stage to the solution of systems of $4 N_{1}$ first-order ordinary differential equations:

$$
\bar{U}_{k}^{\prime}\left(x_{2}\right)=A_{k} \bar{U}_{k}\left(x_{2}\right)+\overline{\widetilde{R}}_{k}\left(x_{2}\right)
$$

where $\bar{U}_{k}$ is global vector of nodal unknowns (subscript “ $(k)$ " corresponds to the number of subdomain $\omega_{k}=\omega_{k}^{d c}$,

$$
\begin{aligned}
& \bar{U}_{k}=\bar{U}_{k}\left(x_{2}\right)=\left[\begin{array}{ll}
\left(\bar{u}_{k}\right)^{T} & \left(\bar{v}_{k}\right)^{T}
\end{array}\right]^{T} ;
\end{aligned}
$$

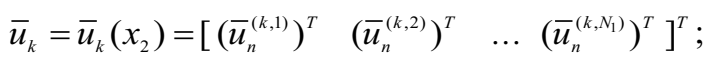

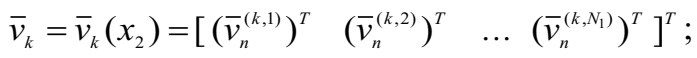

$$
\begin{aligned}
& \bar{u}_{n}^{(k, i)}=\bar{u}_{n}^{(k, i)}\left(x_{2}\right)=\left[\begin{array}{lll}
u_{1}^{(k, i)} & u_{2}^{(k, i)}
\end{array}\right]^{T} ; \bar{v}_{n}^{(k, i)}=\bar{v}_{n}^{(k, i)}\left(x_{2}\right)=\left[\begin{array}{ll}
v_{1}^{(k, i)} & v_{2}^{(k, i)}
\end{array}\right]^{T} ;
\end{aligned}
$$

$A_{k}$ is global matrix of coefficients of order $4 N_{1} ; \overline{\widetilde{R}}_{k}\left(x_{2}\right)$ is the right-side vector of order $4 N_{1}$. Correct analytical solution of $(21)$ is defined by formula

$$
\begin{gathered}
\bar{U}_{k}\left(x_{2}\right)=E_{k}\left(x_{2}\right) \bar{C}_{k}+\bar{S}_{k}\left(x_{2}\right) ; \\
E_{k}\left(x_{2}\right)=\varepsilon_{k}\left(x_{2}-x_{2, k}^{b}\right)-\varepsilon_{k}\left(x_{2}-x_{2, k+1}^{b}\right) ; \quad \bar{S}_{k}\left(x_{2}\right)=\varepsilon_{k}\left(x_{2}\right) * \overline{\widetilde{R}}_{k}\left(x_{2}\right) ;
\end{gathered}
$$

$\varepsilon_{k}\left(x_{2}\right)$ is the fundamental matrix-function of system (20), which is constructed in the special form convenient for problems of structural mechanics [1-5,35]; * is convolution notation; $\bar{C}_{k}$ is the vector of constants of order $4 N_{1}$.

\section{Multilevel approximation model for domain}

Passing to multilevel approximation for domain is described in [3-5] for the simplest case of two-point boundary problem (Fig. 3). In a more general case, this transition is carried out in a similar way, although, of course, also with the use of more complicated formulas. 


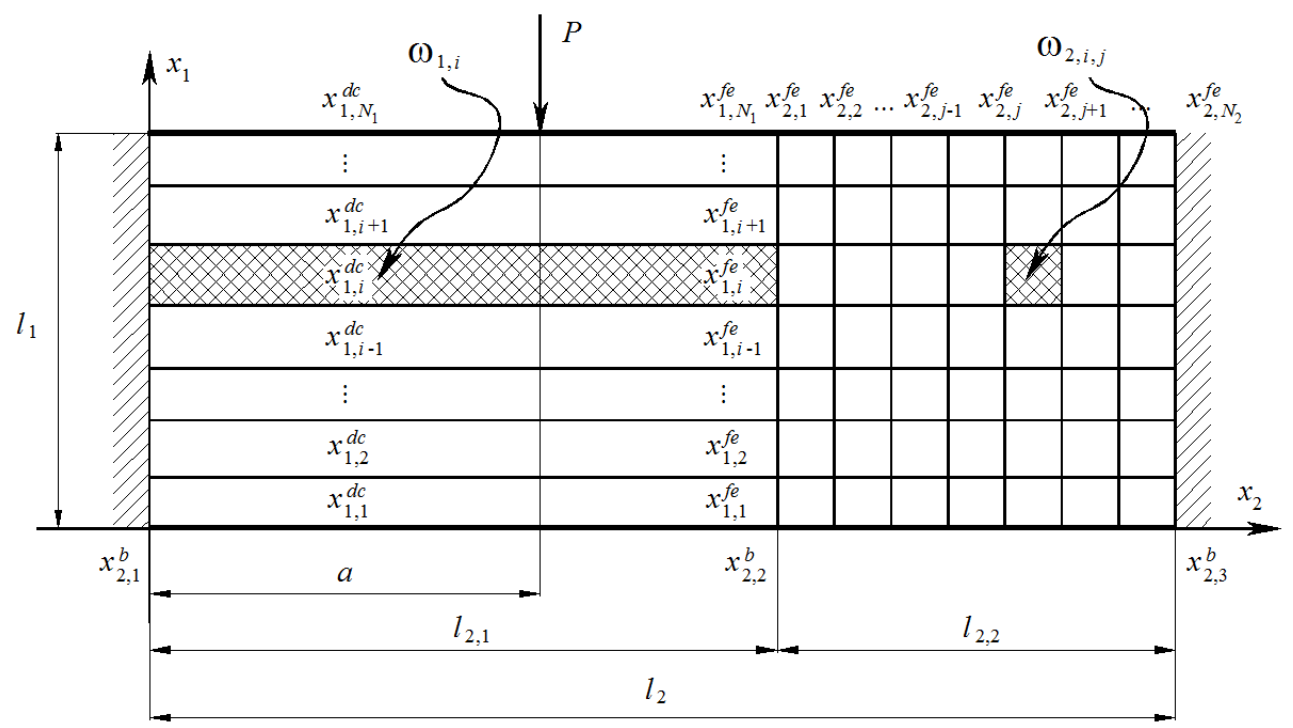

Fig. 3. Two-point boundary problem of static analysis of deep beam [3-5,30].

\section{Software and verification samples}

We should stress that all methods and algorithms considered in this paper have been realized in software. The main purpose of Analysis system CSASA2Dm (DCFEM + FEM) is semianalytical structural analysis (static structural analysis of deep beam within twodimensional theory of elasticity), based on combined application of FEM and DCFEM. Programming environment is Microsoft Visual Studio 2013 Community and Intel Parallel Studio 2017XE (Fortran programming language [16]) with Intel MKL Library [8]. Software is designed for Microsoft Windows 8.1/10.

The Reported study was Funded by Government Program of the Russian Federation "Development of science and technology" (2013-2020) within Program of Fundamental Researches of Ministry of Construction, Housing and Utilities of the Russian Federation and Russian Academy of Architecture and Construction Sciences, the Research Project 7.1.1".

\section{References}

1. P.A. Akimov, Applied Mechanics and Materials, 204-208, 4502-4505 (2012).

2. P.A. Akimov, M.L. Mozgaleva, Applied Mechanics and Materials, 580-583, 28982902 (2014).

3. P.A. Akimov, O.A. Negrozov, Procedia Engineering, 153, 8-15 (2016).

4. P.A. Akimov, O.A. Negrozov, Procedia Engineering, 153, 16-23 (2016).

5. P.A. Akimov, O.A. Negrozov, Procedia Engineering, 153, 24-31 (2016).

6. E.M. Alawadhi, Finite Element Simulations Using ANSYS (CRC Press, 2009).

7. R. Azam, K. Soudki, Cement and Concrete Composites, 34(8), 946-957 (2012).

8. E.J. Barbero, Finite Element Analysis of Composite Materials Using ANSYS (CRC Press, 2013).

9. K.-J. Bathe, Finite Element Procedures (Prentice Hall Inc., 1996). 
10. G. Campione, G. Minafo, Engineering Structures, 41, 294-306 (2012).

11. M.-Y. Cheng, M.-T. Cao, Engineering Applications of Artificial Intelligence, 28, 89-96 (2014).

12. Y.K. Cheung, F.T.K. Au, D.Y. Zheng, Thin-Walled Structures, 32(4), 289-303 (1998).

13. E.H. Dill, The Finite Element Method for Mechanics of Solids with ANSYS Applitions (CRC Press, 2011).

14. A.H. Gandomi, A.H. Alavi, D.M. Shadmehri, M.G. Sahab, Archives of Civil and Mechanical Engineering, 13(3), 354-369 (2013).

15. Y.H. Gedik, H. Nakamura, N. Ueda, M. Kunieda, Procedia Engineering, 14, 29642971 (2011).

16. R.J. Hanson, T. Hopkins, Numerical Computing with Modern Fortran (Applied Mathematics) (SIAM-Society for Industrial and Applied Mathematics, 2013).

17. R.A. Hawileh, T.A. El-Maaddawy, M.Z. Naser, Materials \& Design, 42, 378-387 (2012).

18. M.A.T. Khatab, A.F. Ashour, T. Sheehan, D. Lam, Engineering Structures, 131, 264274 (2017).

19. H.S. Kim, M.S. Lee, Y.S. Shin, Procedia Engineering, 14, 2212-2218 (2011).

20. Y.-B. Leng, X.-B. Song, H.-L. Wang, Journal of Constructional Steel Research, 106, 89-98 (2015).

21. E. Lim, S.-J. Hwang, Engineering Structures, 108, 104-112 (2016).

22. Z. Lin, M. Raoof, Engineering Structures, 17(10), 725-736 (1995).

23. J. Liu, B.I. Mihaylo, Engineering Structures, 112, 81-89 (2016).

24. E. Madenci, I. Guven, The Finite Element Method and Applications in Engineering Using ANSYS (Springer, 2015).

25. I.M. Metwally, HBRS Journal, 13(1), 25-38 (2017).

26. S. Moaveni, Finite Element Analysis: Theory and Application with ANSYS (Prentice Hall, 2014).

27. A.R. Mohamed, M.S. Shoukry, J.M. Saeed, Alexandria Engineering Journal, 53(2), 329-339 (2014).

28. K. Mohamed, A.S. Farghaly, B. Benmokrane, K.W. Neale, Engineering Structures, 137, 145-161 (2017).

29. M. Mohammadhassani, M.Z. Jumaat, M. Jameel, Construction and Building Materials, 30, 265-273 (2012).

30. O.A. Negrozov, P.A. Akimov, I.Yu. Lantsova, Procedia Engineering, 153, 926-932 (2016).

31. M. Pal, S. Deswal, Computers \& Structures, 89(13-14), 1430-1439 (2011).

32. K. Uenaka, H. Tsunokake, Structures, 10, 89-95 (2017).

33. O.C. Zienkiewicz, R.L. Taylor, D.D. Fox, The Finite Element Method for Solid and Structural Mechanics (Butterworth-Heinemann, 2013).

34. O.C. Zienkiewicz, R.L. Taylor, J.Z. Zhu, The Finite Element Method: Its Basis and Fundamentals (Butterworth-Heinemann, 2005).

35. A.B. Zolotov, P.A. Akimov, Proceedings of the International Symposium LSCE 2002 organized by Polish Chapter of IASS (Warsaw, Poland, 2002). 